\title{
ANÁLISIS Y SIMULACIÓN DE TIEMPOS DE ESPERA APLICANDO TEORÍA DE COLAS EN UNA TERMINAL DE TRANSPORTES
}

\author{
ANALYSIS AND SIMULATION OF WAITING TIMES BY APPLYING \\ QUEUING THEORY IN A TRANSPORT TERMINAL
}

\author{
Jorge Enrique Narváez-Gómez ${ }^{1}$, Walter Arley Ordońez-Luna², Nelson Emilio Paz-Ruiz ${ }^{3}$ \\ Corporación Universitaria Comfacauca, Facultad de Ingeniería, Popayán, Cauca, Colombia
}

Recibido: 16/01/2018 • Aprobado: 23/05/2018

\section{RESUMEN}

El objetivo de este trabajo es medir el desempeño en la prestación de servicio del Terminal de Transportes Popayán S.A. (Cauca). Para dicho análisis se tomó el puente festivo correspondiente al 17 de octubre de 2016, siendo este uno de los escenarios en donde se presenta mayor flujo de personas en las diferentes empresas de transporte. La ruta evaluada correspondió a Popayán - Cali para las diferentes empresas que la manejaban. La determinación de esa ruta se basó en los datos estadísticos brindados por la terminal, quien la sitúa entre los primeros lugares en cuanto a afluencia. Mediante el desarrollo y aplicación de la metodología de Poisson para el análisis de líneas de espera, se identificó que la tasa de servicio se distribuye exponencialmente. El servicio de llegada presentó una tendencia Poisson, presentando un solo servidor dentro de la estructura del servicio y fue modelada mediante un modelo matemático tipo $(\mathrm{M} / \mathrm{M} / 1)$. Este modelo permitió encontrar el nivel de desempeño de cada una de las empresas en observación. Finalmente, el estudio determinó que Tax Belalcázar es una de las empresas con mayor eficacia a la hora de prestar el servicio. Los resultados se corroboran mediante la simulación del modelo en el software para ingeniería FlexSim, concluyendo que, en este tipo de escenarios, alrededor de 259 personas no tienen la posibilidad de acceder al servicio.

Palabras clave: distribución, modelos de líneas de espera, simulación.

\section{Abstract}

The objective of this study is to measure the performance in the service provision of the Popayan transport terminal (Cauca). For this analysis the festive bridge corresponding to October 17, 2016 was taken, this being one of the scenarios where there is a greater flow of people in the different transport companies. The route evaluated corresponded to Popayan - Cali for the different companies that handled it. The determination of this route was based on the statistical data provided by the terminal, which ranks it among the first places in terms of

\footnotetext{
1 jorgenarvaez@unicomfacauca.edu.co, orcid.org/0000-0003-3385-5083

${ }^{2}$ walterordonez@unicomfacauca.edu.co, orcid.org/0000-0002-1558-6695

${ }^{3}$ npaz@unicomfacauca.edu.co, orcid.org/0000-0003-4010-4341
} 
affluence. Through the development and application of the Poisson methodology for the analysis of waiting lines, it was identified that the service rate is distributed exponentially. The arrival service presented a Poisson trend, presenting a single server within the structure of the service and was modeled using a mathematical model type (M/M/1). This model allowed to find the level of performance of each of the companies under observation. Finally, the study determined that Taxbelalcazar is one of the most effcient at the time of providing the service. The results are corroborated by simulating the model in FlexSim engineering software, concluding that, in this type of scenario, around 259 people do not have the possibility of accessing the service.

Key words: distribution, simulation, waiting line models.

\section{INTRODUCCIÓN}

Los tiempos de espera, en terminales de transporte, se han hecho tan comunes, que, a la hora de calcular el tiempo necesario para trasladarse de un lugar a otro, es de vital importancia contemplarlos para evitar retrasos en compromisos personales (Rochel Benitez, 2000). En ocasiones es un paréntesis inevitable y molesto que puede tardar más tiempo que el mismo viaje, las líneas de espera varían en función de la ruta y las temporadas del año en las que se elija realizar el viaje (AlmeidaGarcía, 2006).

Para una empresa como Terminal de Transportes Popayán S.A., por la cual transitan alrededor de 502.614 usuarios por mes.

Para el (Terminal de Transportes Popayan, Cauca, Colombia, 2016), es importante elegir un sistema de prestación de servicios acorde con la alta demanda del recurso de transporte, de lo contrario se generará una mala experiencia para el usuario, lo que se verá reflejado en la mala percepción hacia la empresa. Por medio de la aplicación de un modelo matemático, que se rige por medio de parámetros que afectan de manera directa las líneas de espera, la teoría de colas permite dar una estimación de la cantidad de recursos que se deben tener disponibles para cumplir con las expectativas de los usuarios y encontrar un equilibrio entre tiempo de espera, tiempo de servicio y recursos disponibles (Badii, et al., 2011).

\section{Metodología}

La metodología que se utilizó para llevar a cabo la investigación es explicada a continuación:

\subsection{Selección de empresas de transporte a estudiar.}

Actualmente la terminal de Popayán, Cauca, Colombia, cuenta con 21 empresas de transporte de pasajeros habilitadas por el Ministerio de Transporte, de las cuáles se seleccionaron seis empresas, con el objetivo de poder realizar una comparación entre líneas de espera, para lo cual se tuvieron en cuenta las de mayor cantidad de usuarios con destino a la ciudad de Cali, Valle del Cauca, Colombia, entre las cuales se encuentran Tax Belalcázar, Trans Timbio, Expreso Palmira, Velotax, Rápido Tambo y Puerto Tejada.

\subsection{Recopilación de información}

La precisión en la medición de tiempos entre llegadas de usuarios y los tiempos que demoran los servidores en prestar el servicio de recepción a los usuarios, es uno de los factores más importantes a la hora de alimentar los modelos matemáticos (Singer, Donoso, \& Scheller-Wolf, 2008), para el análisis de líneas de espera, la llegada desmedida de usuarios al terminal de transportes de Popayán dificulta en gran medida la recolección de la información; con el fin de facilitar este proceso se crearon dos formatos de recolección de información. 


\subsection{Análisis de datos}

En esta etapa, se realizan las siguientes actividades:

- Se cargaron los datos recolectados sobre las empresas seleccionadas.

- Estandarización de la información con el fin de verificar que estén acorde a las distribuciones presentes en los sistemas de colas.

- Se tomó el número de interacciones con respecto a los tiempos, para determinar la tasa de llegada.

\subsection{Arquetipo del proceso y demora del sistema de lineas de espera}

Por medio de la Figura 1. se evidencia como se encuentra actualmente estructurado el sistema de prestación de servicios, una tasa de llegada de usuarios que se destruye de manera Poisson, la generación de llegadas acumuladas como efecto de la alta demanda del servicio y una tasa de atención que se distribuye de manera exponencial (Hillier, Lieberman, \& Osuna, 1997) (Martínez, 2009).

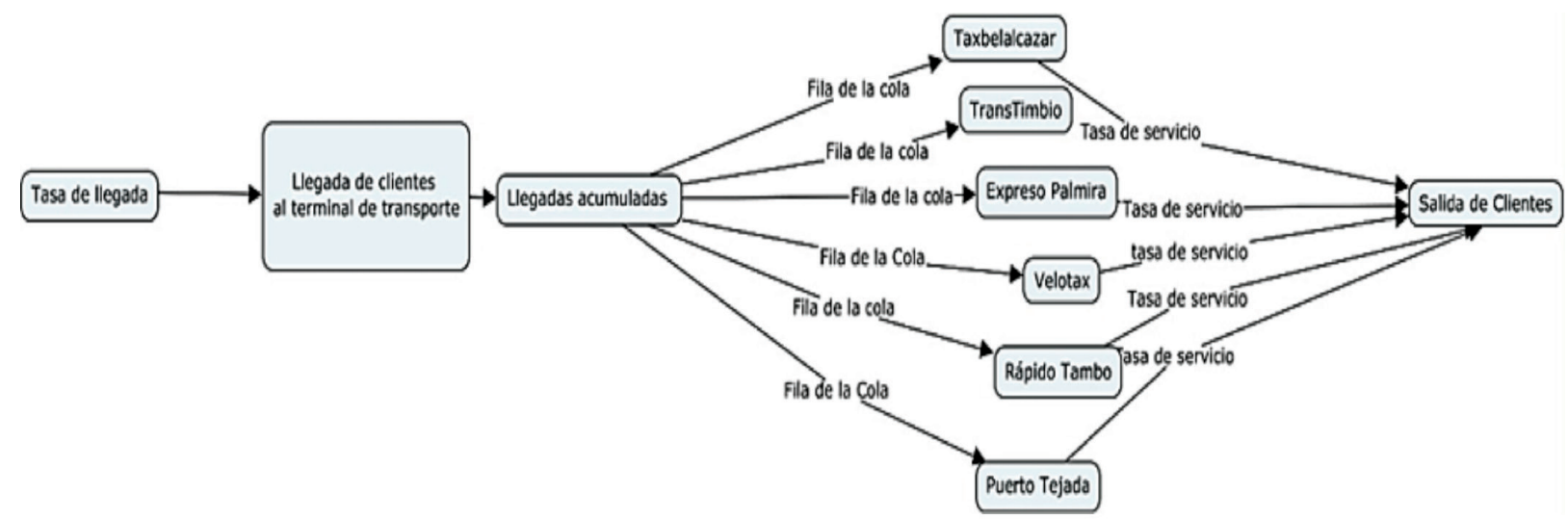

Fig. 1. Arquetipo del sistema de líneas de esperas

\section{Resultados}

Tomando como referencia la información recopilada sobre los tiempos de espera y durante el servicio en el terminal de transportes de Popayán, en las empresas ya mencionadas anteriormente, se procede a aplicar la metodología de líneas de espera o teoría de colas.

\subsection{Calculo de tasas}

Con base en los tiempos tomados, referentes al sistema de servicio prestado por las empresas, se calcularon las tasas de llegada $\lambda$, que representan la totalidad del tiempo sobre el promedio de entrada y tasas de servicios $\mu$ : $(\lambda=$ clientes/tiempo, $\mu=$ tiempo/clientes $)$.

- Tax Belalcázar: $\lambda=84,75$ personas/hora, $\lambda=1,41$ personas/minuto $1 \lambda=0,01$ y $\mu=0,015$ horas/persona, $\mu=0,907$ minutos/persona.
- Expreso Palmira: $\lambda=65,63$ personas/hora, $\lambda=1,09$ personas/minuto $1 \lambda=0,015$ y $\mu=0,02$ horas/persona $\mu=1,197$ minutos/persona.

- Trans Timbio: $\lambda=79$ personas $/$ hora, $\lambda=1,31$ personas/minuto $1 \lambda=0,01$ y $\mu=0,0143$ horas/persona $\mu$ $=0,86 \mathrm{minutos} /$ persona.

- Velotax: $\lambda=56,75$ personas/hora, $\lambda=0,945$ personas/minuto $1 \lambda=0,018$ у $\mu=0,015$ horas/persona $\mu$ $=0,889 \mathrm{minutos} /$ persona .

- Rápido Tambo: $\lambda=50,75$ personas/hora, $\lambda=0,845$ personas/minuto $1 \lambda=0,019$ y $\mu=0,018$ horas/persona $\mu=1,058$ minutos/persona.

- Puerto Tejada: $\lambda=46,4$ personas $/$ hora, $\lambda=0,77$ personas/minuto $1 \lambda=0,02$ y $\mu=0,017$ horas/persona $\mu$ $=1,034 \mathrm{minutos} /$ persona. 


\subsection{Aplicación de ecuaciones de líneas de Espera} de Poisson

Para evaluar y analizar la eficacia en la prestación del servicio, se aplican las siguientes ecuaciones propuestas por Poisson (Alcázar \& García , 2012) (Gómez, 2011) para el análisis de modelo de líneas de espera:

$\boldsymbol{\rho}=\frac{\lambda}{\mu} \begin{aligned} & \text { Proporción del tiempo que el sistema está } \\ & \text { ocupado o la probabilidad de que esté ocu- }\end{aligned}$ pado.

$\mathbf{P}(\mathbf{0})=\mathbf{1}-\boldsymbol{\rho}$ Probabilidad de encontrar el sistema vacío.

$\mathbf{L q}=\frac{\boldsymbol{\rho}^{\mathbf{2}}}{\mathbf{1 - \boldsymbol { \rho }}} \begin{aligned} & \text { Número de unidades a encontrar } \\ & \text { en la cola. }\end{aligned}$

$$
\mathbf{L q}=\frac{\boldsymbol{\rho}^{\mathbf{2}}}{\mathbf{1 - \boldsymbol { \rho }}}=\boldsymbol{L q}+\boldsymbol{\rho} \quad \begin{aligned}
& \text { Número de unidades } \\
& \text { a encontrar en el sistema }
\end{aligned}
$$

$\mathbf{W q}=\frac{\mathbf{L q}}{\lambda}$ Tiempo de espera en la cola.

$\mathbf{W}=\frac{1}{\mu-\lambda}$ Tiempo de espera en el sistema.

\section{ANÁlisis de datos}

Teniendo en cuenta el comportamiento de los datos recopilados se analizarán los datos globales, ósea en la totalidad del puente festivo y un análisis detallado para determinar horas de menos dificultad a la hora de adquirir el servicio.

TABla 1

\begin{tabular}{|c|c|c|c|c|}
\hline \multicolumn{5}{|c|}{ Cálculo De Tasas } \\
\hline \multirow{2}{*}{ Empresas } & \multicolumn{2}{|c|}{ Llegada } & \multicolumn{2}{|c|}{ Atención } \\
\hline & Pesonas/Hora & Personas/Minuto & Hora/Persona & Minuto/Persona \\
\hline TaxBelalcazar & 85 & 2 & 0,015 & 0,907 \\
\hline Expreso Palmira & 66 & 2 & 0,02 & 1,197 \\
\hline Transtimbio & 79 & 2 & 0,0143 & 0,86 \\
\hline Velotax & 57 & 1 & 0,015 & 0,889 \\
\hline Rápido Tambo & 51 & 2 & 0,018 & 1,058 \\
\hline Puerto Tejada & 47 & 1 & 0,017 & 1,034 \\
\hline
\end{tabular}

Tasa de llegada y tasa de servicio

Fuente: autores

De acuerdo a los valores calculados de las tasas de llegada $(\lambda)$, y las tasas de servicio $(\mu)$ en la Tabla 1 , se evidenció que Tax Belalcázar sobresale entre las seis empresas estudiadas, debido a que presenta la tercera tasa de atención más rápida con $\mu=0,907$ minutos/ persona, a pesar de que también es quien mayor tasa de llegada genera con $\lambda=85$ personas/hora. 


\subsection{Porcentaje de utilización del servicio}

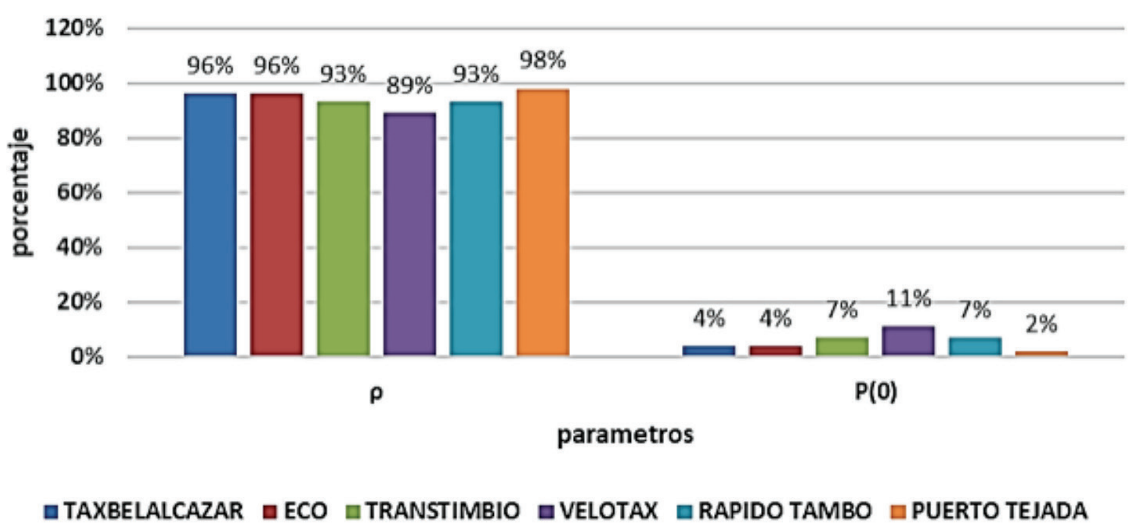

Fig. 1. Probabilidad de encontrar vacío el sistema

Fuente: autores

Teniendo en cuenta la globalización de los datos y que estos se tomaron en un puente festivo, se identificó que todas las empresas que fueron de interés para la investigación, presentan un alto porcentaje de ocupación, lo que hace que la probabilidad de encontrar el servicio vacío se reduzca considerablemente.
Como se puede observar en la figura 2, la empresa que representa mayor oportunidad de encontrar el sistema vacío es Velotax con un $11 \%$, la empresa con mayor ocupación del sistema fue Puerto Tejada con un $98 \%$.

\subsection{Porcentaje de utilización del servicio por horas}

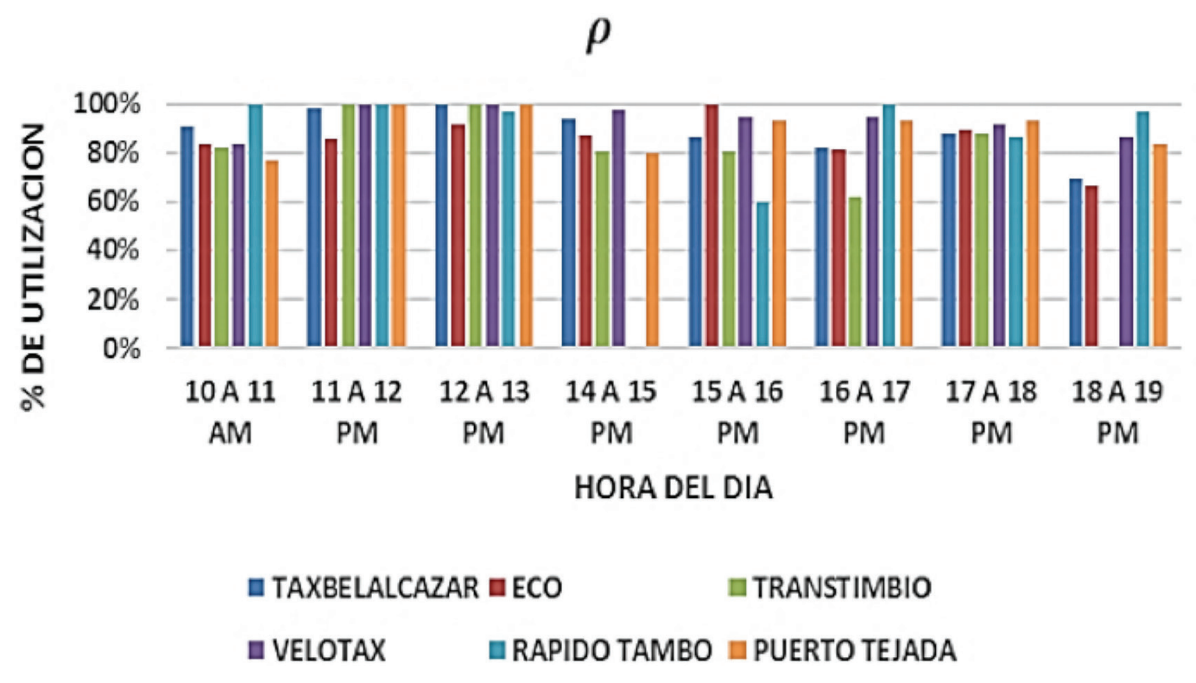

Fig. 2. Probabilidad de encontrar vacío el sistema por horas

Fuente: autores

Como se observa en la figura 3, las probabilidades de encontrar el sistema vacío son muy bajas a lo largo del día, sin embargo, antes de las 10 de la mañana y entre las tres de la tarde y las cinco de la tarde se registra unos intervalos en los cuales el tiempo de espera para la obtención del servicio es menor. 


\subsection{Tiempo de espera para la obtención del servicio}

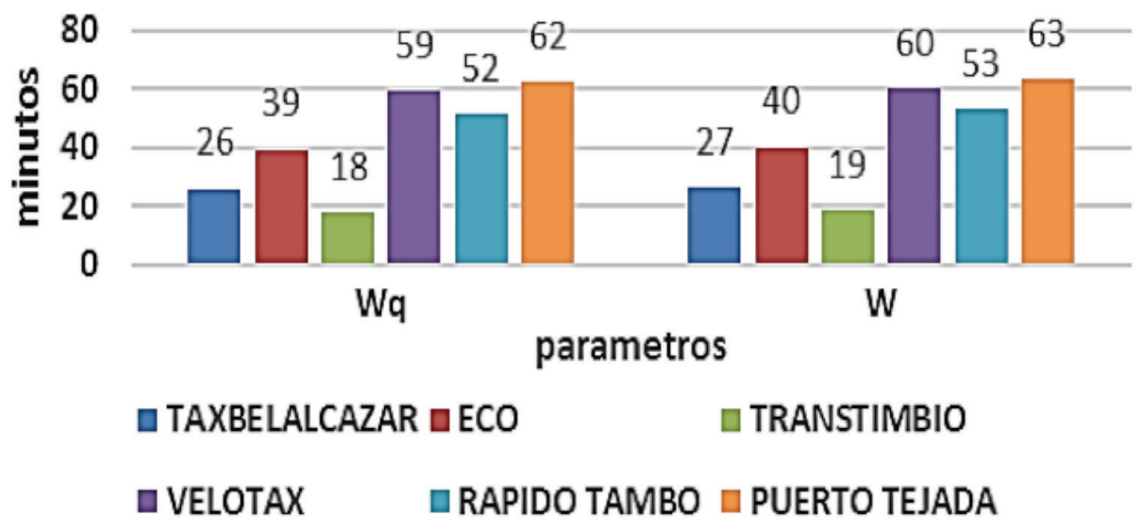

Fig. 3. Tiempo de espera en el servicio y cola Fuente: autores

Teniendo en cuenta la elevada demanda que se presenta durante un puente festivo y teniendo presente que los días lunes y viernes es cuando más se utiliza el servicio, se calculó el tiempo promedio de espera para obtener el servicio, en el cual, la empresa Puerto Tejada, con 62 minutos en promedio, es quien presenta el mayor tiempo de espera y esto es apenas lógico ya que también es quien presenta el mayor porcentaje de tiempo de ocupación. En cuanto a Trans Timbio, que es una de las empresas que mayor porcentaje de ocupación presenta, también es de las de menor cantidad de tiempo de espera para la obtención del servicio.

\subsection{Tiempo de espera para la obtención del servicio por horas}

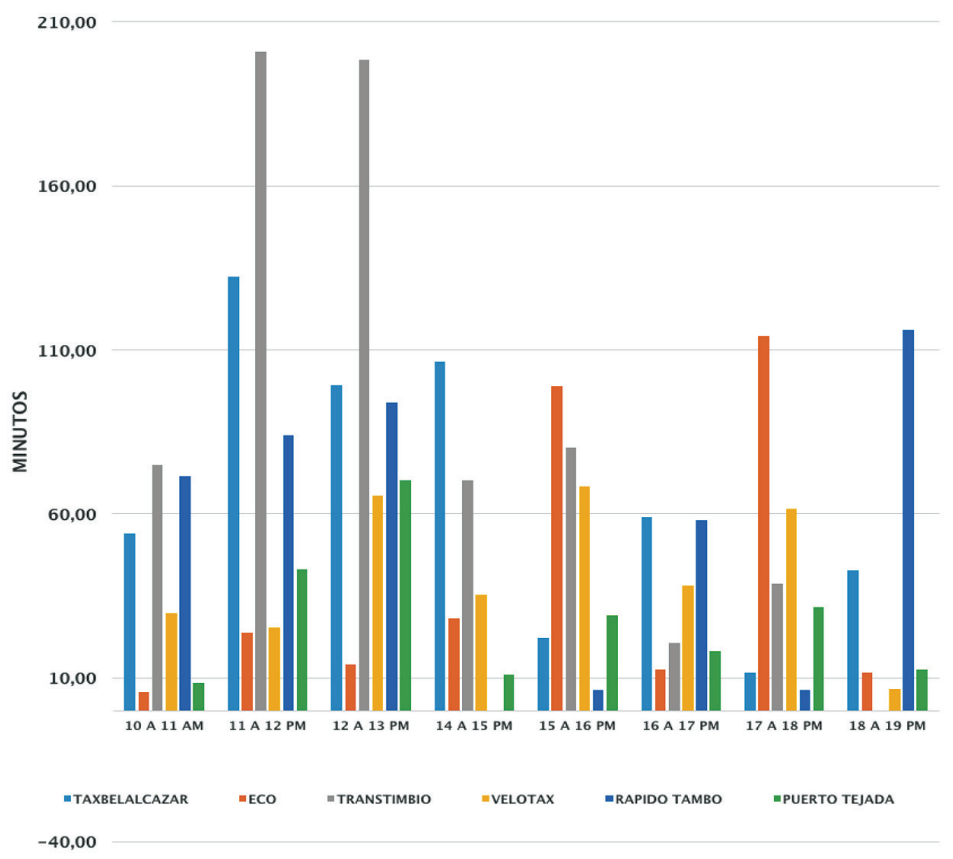

Fig. 4. Tiempo de espera por horas Fuente: autores 
Como se observa en la Figura 5, el tiempo a esperar podría elevarse hasta 200 minutos, algo que podría entenderse como inconcebible, pero haciendo referencia a que este fue un puente festivo es muy frecuente encontrar esperas tan prolongadas, los intervalos donde se presentan menores esperas están considerados antes de las 10 de la mañana y entre las tres de la tarde y las siete de la noche.

\section{Simulación}

\subsection{Costos asociados a la espera}

Se planteó conocer cuál era el costo que conllevaba la espera o que distintos clientes deserten de la fila por falta de servicio, cupos limitados y cansancio, entre otras situaciones.

Por medio de los parámetros y variables calculados, se construyó el modelo de líneas de espera en la herramienta de simulación para ingeniería FlexSim, lo cual arrojó una cantidad promedio de 259 personas que estaban dejando de atenderse por día durante este puente festivo, para estimar un valor monetario para esta suma de personas, se planteó el supuesto que todos estos usuarios tenían como destino la ciudad de Cali, sabiendo que el transporte hasta esta ciudad tiene un costo de $\$ 25.000$ por persona, el costo total que tendrá la pérdida sería de $\$ 6.475 .000$. por día.

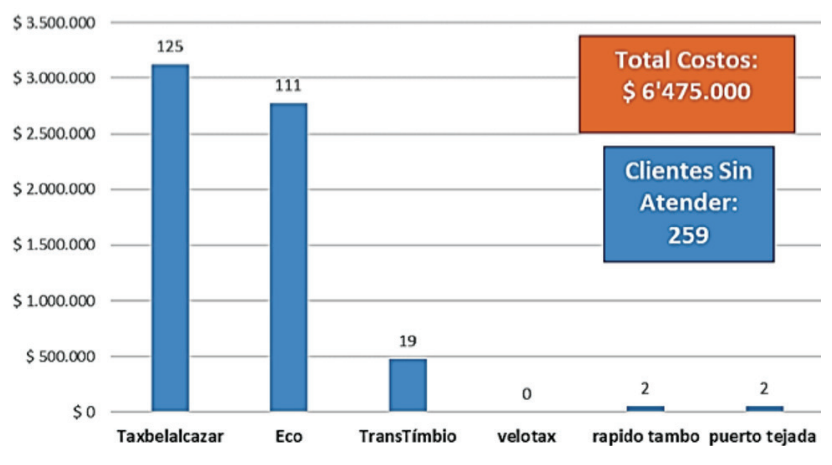

Fig. 5. Costos asociados a la espera Fuente: autores

\subsection{Comparación del modelo teórico con la simulación}

Una vez definidas las variables del modelo de colas en el software de simulación, se plantean los diferentes escenarios para cada una de las empresas de servicio mencionadas en el estudio y se procedió a correr la simulación.

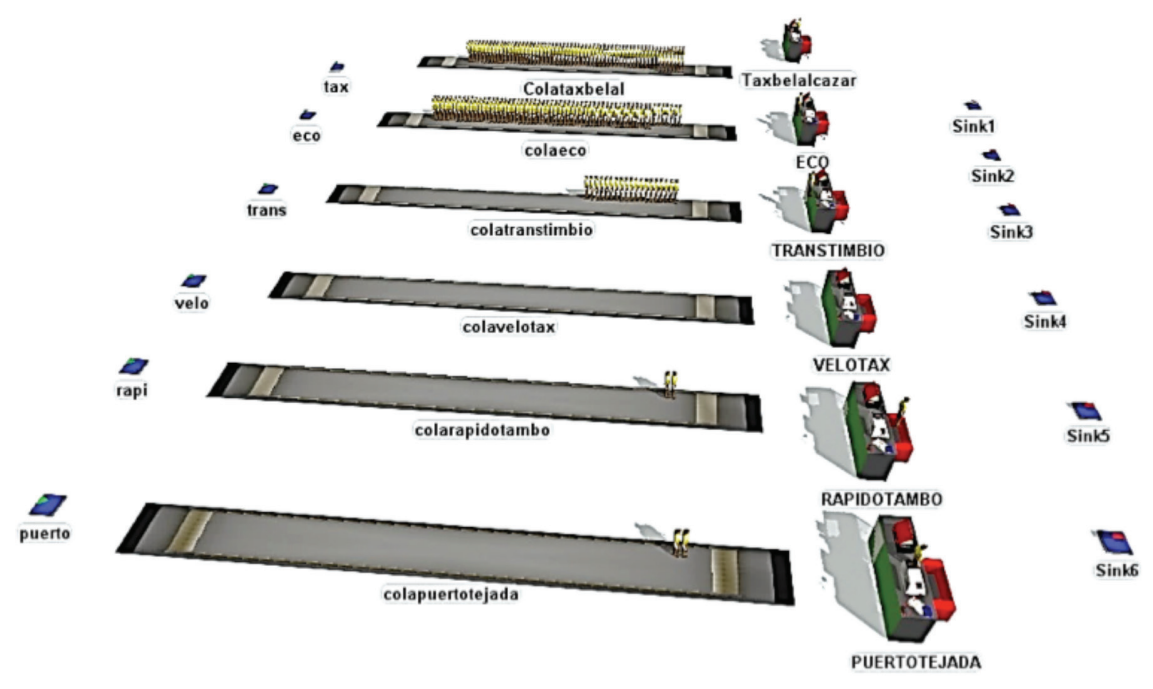

Fig. 6. Simulación de modelo de colas M/M/1 en software FlexSim

Al comparar las cifras de las medidas de desempeño del modelo teórico con las encontradas en el modelo simulado (Tabla 1), se evidencia un comportamiento similar, esto se corrobora gracias a que el margen de 
diferencia entre los resultados de los dos modelos (teórico y simulado), está alrededor del 6\% para el parámetro P y 5.63\% para el parámetro Wq (ver figuras 3 y 4$)$.

TABla 2

Resultados parámetro P software FlexSim

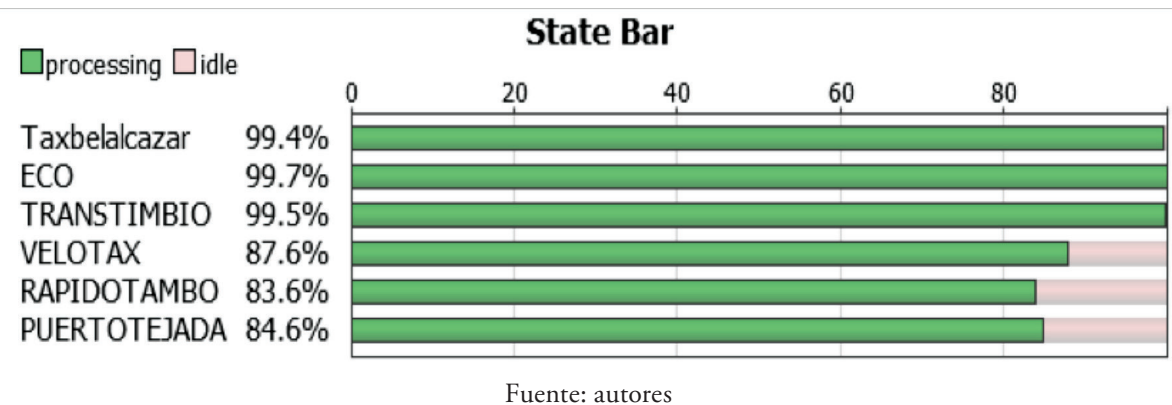

Tabla 3

Comparación de resultados. Elaboración propia

\begin{tabular}{|c|c|c|c|c|c|c|}
\hline \multirow{2}{*}{ Empresas } & \multicolumn{2}{|c|}{ Parámetro teórico } & \multicolumn{2}{|c|}{ Parámetro simulación } & \multicolumn{2}{|c|}{$\begin{array}{l}\text { \% Diferencia teórico } \\
\text { vs simulación }\end{array}$} \\
\hline & $\mathbf{P}$ & Wq & $\mathbf{p}$ & Wq & $\mathbf{P}$ & Wq \\
\hline Tax Belalcázar & $96 \%$ & 26 & $99,40 \%$ & 36,12 & $3 \%$ & $7,80 \%$ \\
\hline Eco & $96 \%$ & 39 & $99,70 \%$ & 43,85 & $4 \%$ & $6,60 \%$ \\
\hline Trans Timbío & $93 \%$ & 18 & $99,50 \%$ & 9,53 & $6 \%$ & $2,30 \%$ \\
\hline Velotax & $89 \%$ & 59 & $87,60 \%$ & 23,5 & $1 \%$ & $11,30 \%$ \\
\hline Rápido Tambo & $93 \%$ & 52 & $83,60 \%$ & 45,7 & $9 \%$ & $2,50 \%$ \\
\hline Puerto Tejada & $98 \%$ & 62 & $84,60 \%$ & 42 & $13 \%$ & $3,30 \%$ \\
\hline
\end{tabular}

Fuente: los autores

\section{Observaciones y Recomendaciones}

- Realizar un análisis más detallado para determinar qué tan factible es poner en funcionamiento un segundo servidor, para temporadas en las que las tasas de llegada superan la capacidad de servicio.

- Uno de los problemas, que a simple vista presentaban todas las empresas, era la falta de información en cuanto a destinos y hora de salida; esto generaba la acumulación de más de un cliente en el servidor interrumpiendo la adecuada prestación del servicio, de igual forma ralentizaba la venta de los tiquetes por lo cual se recomienda la instalación de pantallas que permitan observar a los clientes las rutas y horas en las cuales prefieren viajar.

- La falta de control en algunas empresas ralentizaba la venta de tiquetes generando demora en el tiempo de servicio, para lo cual es recomendable aplicar el uso de tecnologías que permitan controlar la capacidad de cada vehículo y además crear una plataforma virtual que facilite la compra de pasajes. 


\section{Conclusiones}

- Es importante aclarar que para este caso se tomó una muestra de seis empresas de transporte de las 25 empresas que están habilitadas por el Ministerio de Transporte para prestar este servicio en la terminal de Popayán. Si se tomara como referencia la totalidad de empresas, el costo se elevaría considerablemente.

- En la tasa de servicios se presentaron datos gobernados por tiempos con alta variabilidad, se denotaron tiempos atípicos que son generados en la mayoría de los casos por una ausencia de cultura a la hora de utilizar el servicio, tales como casos de regateo como comúnmente se le conoce, sistemas ineficaces en la venta de tiquetes, los cuales hacen que los tiempos e incrementen de gran manera.

- Los factores que más inciden en el desempeño de las redes de colas son las distribuciones de probabilidad en la tasa de llegada, por esto se presenta un gran desborde de las filas o de los clientes, y lo que sucede es que para las seis empresas en la mayor cantidad de tiempo la tasa de llegada superó la tasa de servicio y justo en ese momento se pierde el control y los tiempos se ven afectados.

- Uno de los problemas, que a simple vista presentaban todas las empresas, era la falta de información en cuanto a destinos y hora de salida; esto generaba la acumulación de más de un cliente en el servidor interrumpiendo la adecuada prestación, de igual forma ralentizaba la venta de los mismos por lo cual se recomienda la instalación de pantallas que permitan observar a los clientes las rutas y horas en las cuales prefieren viajar.

- La falta de control en algunas empresas ralentizaba la venta de tiquetes generando demora en el tiempo de servicio, para lo cual es recomendable aplicar el uso de tecnologías que permitan controlar la capacidad de cada vehículo y además crear una plataforma virtual que facilite la compra de pasajes.

\section{REFERENCIAS}

Alcázar, L. F. \& García, C. A. (2012). Modelación de línea en espera para el Centro Integral de Atención al Cliente Norte (CIAC Calle 83), de EPM-Bogotá SA ESP. Bogotá: Universidad de la Sabana.

Almeida-García, F. (2015). Tipología de visitante turístico y satisfacción de la experiencia turística en Santiago de Compostela. Baetica, 28(1). Recuperado en: https://riuma.uma.es/xmlui/ handle/10630/6653

Badii, M. H., Guillen, A., Cerna, E. \& Landeros, J. (2011). Dispersión espacial: el prerrequisito esencial para el muestreo. Daena: International Journal of Good Conscience, 6(1), 40-71. Recuperado en: http://www.spentamexico.org/v6-n1/40a71.pdf

Gómez, F. A. (2011). Aplicación de teoría de colas en una entidad financiera: herramienta para el mejoramiento de los procesos de atención al cliente. Revista Universidad EAFIT, 44(150), 51-63. Recuperado en: http://publicaciones.eafit.edu.co/index.php/ revista-universidad-eafit/article/view/154

Hillier, F. S., Lieberman, G. J. \& Osuna, M. A. (1997). Introducción a la investigación de operaciones (Vol. 1). México: McGraw-Hill.

Martínez, C. E. (2009). Análisis de redes de colas modeladas con tiempos entre llegadas exponenciales e híper erlang para la asignación eficiente de los recursos. Tesis doctoral. Facultad de Ingeniería, Bogotá: Universidad Javeriana.

Rochel Benítez, J. J. (2000). Factores determinantes de la demanda de transporte aéreo y modelos de previsión. Boletín económico de ICE, (2652), 41-48.

Singer, M., Donoso, P. \& Scheller-Wolf, A. (2008). Una introducción a la teoría de colas aplicada a la gestión de servicios. Abante, 11, 93-120.

Terminal de Transportes Popayán S. A. (2016). Estadísticas de pasajeros por mes, año 2016. Popayán, Colombia. 
